Pacific Journal of Mathematic 


\title{
EXISTENCE AND ADJOINT THEOREMS FOR LINEAR STOCHASTIC DIFFERENTIAL EQUATIONS
}

\author{
VIRGINIA M. WARFIELD
}

\begin{abstract}
This paper contains three theorems on linear stochastic differential equations, where the differential equations are given in terms of McShane's first and second order related integrals. The first, which is modelled on the classical Picard Theorem, concerns the existence of solutions, the second gives boundedness of their moments, and the third provides them with adjoints. The Adjoint Theorem has the interesting property that its formulation requires the second order integral even when the original differential equation involves only the more standard first order integral.
\end{abstract}

To guarantee the existence of the belated integrals (as defined in [4]), we have universally the following hypotheses:

(1) $(\Omega, \mathscr{A}, P)$ is a probability triple, where $\mathscr{A}$ is a $\sigma$-algebra of subsets of $\Omega$.

(2) $\left(\mathscr{F}_{t}: 0 \leqq t \leqq T\right)$ is a family of $\sigma$-subalgebras of $\mathscr{A}$ such if $0 \leqq s \leqq t \leqq T$, then $\mathscr{F}_{s} \subseteq \mathscr{F}_{t}$.

(3) $z^{\rho}(t, \omega),(\rho=1, \cdots, r)$ are stochastic processes on $[0, T]$, and if $t \in[0, T]$, then $z^{\rho}(t, \omega)$ is $\mathscr{F}_{t}$-measurable.

(4) There exist real numbers $K_{1}, K_{2}, K_{4}$ such that if $0 \leqq s \leqq$ $t \leqq T$, then

$$
\begin{aligned}
\left|E\left(\left[z^{\rho}(t)-z^{\rho}(s)\right] \mid \mathscr{F}_{8}\right)\right| \leqq K_{1}(t-s) & \text { a.s. } \\
E\left(\left[z^{\rho}(t)-z^{\rho}(s)\right]^{c} \mid \mathscr{F}_{8}\right) \leqq K_{c}(t-s) & (c=2,4) \quad \text { a.s. }
\end{aligned}
$$

Also universally, we use the term $L_{2}$-norm and the notation \| \| to mean $L_{2}$-norm with respect to expectation.

Under these hypotheses, an application of Picard's method enables us to prove the following theorem:

THEOREM 1. Suppose $y^{i}(\omega)(i=1, \cdots, n)$ is $\mathscr{F}_{0}$-measurable and $c_{\rho \alpha}^{i}(t, \omega)$ and $C_{\rho \alpha \alpha}^{i}(t, \omega)(i=1, \cdots, n ; \rho, \sigma=1, \cdots, r)$ are bounded stochastic processes continuous in probability a.e. on $[0, T]$, and such that $c_{\rho \alpha}^{i}(t, \cdot)$ and $C_{\rho \sigma \alpha}^{i}(t, \cdot)$ are $\mathscr{F}_{t}$-measurable. Then the following system of equations has a solution

$$
\begin{aligned}
X^{i}(t, \omega)= & y^{i}(\omega)+\int_{0}^{t} \sum_{\rho, \alpha} c_{\rho \alpha}^{i}(s, \omega) X^{\alpha}(s, \omega) d z^{\rho} \\
& +\int_{0}^{t} \sum_{\rho, \sigma, \alpha} C_{\rho \sigma \alpha}^{i}(s, \omega) X^{\alpha}(s, \omega) d z^{\rho} d z^{\sigma} .
\end{aligned}
$$


Furthermore, the solution is continuous in $L_{2}$-norm and is unique in the sense that if $X(t, \omega)$ and $Y(t, \omega)$ are two solutions of the system with the same initial distribution $y(\omega)$, then for all $t \in[0, T]$,

$$
\|X(t, \omega)-Y(t, \omega)\|=0 \text {. }
$$

If one ignores the second integral (the second order term) in $(*)$, then the equation bears some resemblance to the equation discussed in $\S 3.2$ of [2]:

$$
d x=e(x) d b+f(x) d t .
$$

Differences are that his integration is Itô integration-i.e., $b$ is Brownian motion-and his coefficients $e$ and $f$ need not be linear, but cannot be stochastic. A further apparent difference is the result of the following device:

Device. Any equation of the form

$$
x^{i}(s)=\int_{a}^{s} f^{i}(t, x) d t+\int_{a}^{s} \sum_{\rho=1}^{r} g_{\rho}^{i}(t, x) d z^{\rho}+\int_{a}^{s} \sum_{\rho, \sigma=1}^{r} G_{\rho, o}^{i}(t, x) d z^{\rho} d z^{\sigma}
$$

can be written in the form

$$
x^{i}(s)=\int_{a}^{s} \sum_{\rho=1}^{r+1} g_{\rho}^{i}(t, x) d z^{\rho}+\int_{a}^{s} \sum_{\rho, \sigma=1}^{r} G_{\rho_{o}}^{i}(t, x) d z^{\rho} d z^{\sigma} .
$$

This we do by defining $z_{\rho}^{r+1}(t)$ to be equal to $t$ for all $t \in[a, b], g_{r+1}^{i}(t, x)$ to be $f^{i}(t, x)$, and $G_{r+1, \sigma}^{i}(t, x)$ and $G_{\rho, r+1}^{i}(t, x)$ to be zero for $\rho, \sigma=$ $i, \cdots, r ; t \in[a, b]$.

Consequently, writing out the terms involving integration with respect to $t$ is an unnecessary complication, so our theorems are stated without them.

McShane also has several theorems on existence of solutions for the equation with nonlinear coefficients (see, e.g., [4]), but in all cases the coefficients must be real-valued functions rather than random variables.

The remaining theorems require two additional hypotheses:

(5) $\lim _{t-s \rightarrow 0}$ ess. $\sup E\left(\left[z^{o}(t)-z^{\rho}(s)\right]^{c} \mid \mathscr{F}_{s}\right) /(t-s)^{2}=0$ uniformly on $[a, b]$ for $c=6,8$.

(6) $\quad A_{h \rho}^{i}(t, \omega), B_{h \rho o}^{i}(t, \omega)(i, h=1, \cdots, n ; \rho, \sigma=1, \cdots, r)$ are processes adapted to $\mathscr{F}$ which are bounded on $[a, b]$ for a.a. $\omega$, continuous in probability on $[a, b]$, and have a.s. continuous sample paths.

We also make heavy use of the following two equations:

I.

$$
\begin{aligned}
x^{i}(t, \omega)= & x^{i}(a, \omega)+\int_{a}^{t} \sum_{h, \rho} A_{h \rho}^{i}(s, \omega) x^{h}(s, \omega) d z^{\rho} \\
& +\int_{a}^{t} \sum_{h, \rho, \sigma} B_{h \rho \sigma}^{i}(s, \omega) x^{h}(s, \omega) d z^{\rho} d z^{\sigma}
\end{aligned}
$$


II. $y^{i}(t, \omega)=y^{i}(a, \omega)-\int_{a}^{t} \sum_{h, \rho} A_{i \rho}^{h}(s, \omega) y^{h}(s, \omega) d z^{\rho}$

$$
+\int_{a}^{t} \sum_{h \rho \sigma}\left[-B_{i \rho \sigma}^{h}(s, \omega)+\sum_{k=1}^{n} A_{k o}^{h}(s, \omega) A_{i \rho}^{k}(s, \omega)\right] y^{h}(s, \omega) d z^{\rho} d z^{\sigma} .
$$

Theorem 2. Assume hypotheses (1)-(6) hold. Then if $x(t, \omega)$ is a solution of equation $I$ with $x(a, \omega)$ bounded for a.a. $\omega$, then there is a constant $R$ depending only on the bounds for $A_{h \rho}^{i}$ and $B_{h \rho \sigma}^{i}$ and on the constants $K_{1}, K_{2}, K_{4}$ in hypothesis 4 such that for all $t \in[a, b]$

$$
E\left\{\left[x^{i}(t, \omega)\right]^{4}\right\} \leqq R E\left\{\max _{i}\left[x^{i}(\alpha, \omega)\right]^{4}\right\} .
$$

An immediate generalization of this theorem is the following:

Corollary. For any $q$, if we add on the hypothesis that

$$
\lim _{t \rightarrow s} \operatorname{ess.} \sup \frac{E\left(\left[z^{o}(t)-z^{\rho}(s)\right]^{2 q} \mid \mathscr{F}_{s}\right)}{(t-s)^{2}}=0 \text { uniformly on }[a, b],
$$

we can find a constant $R$ such that

$$
E\left\{\left[x^{i}(t, \omega)\right]^{q}\right\} \leqq R E\left\{\max \left[x_{i}(\alpha, \omega)\right]^{q}\right\} .
$$

Theorem 3. (Adjoint Theorem): Assume that hypotheses (1)-(6) hold and that $x(a, \omega)$ and $y(a, \omega)$ are bounded for a.a. $\omega$. Suppose $x^{i}(t, \omega)$ is a solution of equation $I$. Then there exists a stochastic process $y^{i}(t, \omega)$ such that for any $t \in[a, b]$

$$
\sum_{i=1}^{n} x^{i}(t, \omega) y^{i}(t, \omega)=\sum_{i=1}^{n} x^{i}(a, \omega) y^{i}(\alpha, \omega) \text { a.s. }
$$

$y^{i}(t, \omega)$ can be obtained as a solution of II.

Note that even in the event that $x(t, \omega)$ has no second order integral terms its adjoint must have them.

The Adjoint Theorem is used in [8] to derive a stochastic version of the Pontryagin Maximum Principle. If one tries to follow the derivation in [7] making everything stochastic as one goes, one is led to an expression involving integration with respect to backwards Brownian motion (cf. [1]). Since integration with respect to backwards Brownian motion is undefined, this creates an unpleasant situation. Our Adjoint Theorem allows us to use the basic structure of Pontryagin's derivation to achieve a set of Lagrange multipliers without getting involved with backwards Brownian motion. There is, however, the drawback that instead of an initial condition we must use a terminal condition. 
In order to prove Theorem I, we need the following result from [4]:

THEOREM 3.1. Suppose hypotheses (1)-(4) hold for $z^{1}$ and $z^{2}$ and suppose $f(t, \omega)$ has the following property:

$I_{2}:$ for all $t \in[a, b], f(t, \omega) \in L_{2}(\Omega, \mathscr{A}, P) ;\|f(t)\|_{2}$ is bounded on $[a, b]$ and $f$ is a.e. (w.r.t. Lebesgue measure) continuous in $L_{2}$-norm on $[a, b]$. Then the integrals

$$
\int_{a}^{o} f(t) d z^{1} \text { and } \int_{a}^{b} f(t) d z^{1} d z^{2} \text { exist. }
$$

For Theorems 1 and 2 we need to use Cauchy polygons and their relationship to stochastic integrals. Our definition, which follows, is parallel to that of McShane in [5]. It differs very slightly from McShane's more recent version as it appears in [6] or that of Maruyama in [3].

Let $\pi$ denote an ordered $(m+1)$-tuple of points $\left(t_{1}, \cdots, t_{m+1}\right)$ with $a=t_{1}<t_{2}<\cdots<t_{m+1}=b$. We define

$$
\operatorname{mesh} \pi=\max \left[t_{h+1}-t_{h}: h=i, \cdots, m\right] \text {. }
$$

Then to the differential equation

$$
\begin{aligned}
x^{i}(t, \omega)= & y^{i}(\omega)+\int_{a}^{t} \sum_{\rho \alpha} c_{\rho \alpha}^{i}(t, \omega) x^{\alpha}(t, \omega) d z^{\rho} \\
& +\int_{a}^{t} \sum_{\sigma \rho \alpha} C_{\rho \sigma \alpha}^{i}(t, \omega) x^{\alpha}(t, \omega) d z^{\rho} d z^{\sigma},
\end{aligned}
$$

there corresponds a Cauchy polygon $x_{\pi}(t, \omega)$ defined by successive steps as follows:

(i) $x^{i}(\alpha, \omega)=y^{i}(\omega)$

(ii) if $x_{\pi}$ has been defined for $t$ on $\left[a, t_{h}\right]$, we define

$$
\begin{aligned}
x^{i}\left(t_{h+1}, \omega\right)= & x^{i}\left(t_{h}, \omega\right)+\sum_{\rho \alpha} c_{\rho \alpha}^{i}\left(t_{h}, \omega\right) x_{\pi}^{\alpha}\left(t_{h}, \omega\right)\left[z^{\rho}\left(t_{h+1}\right)-z^{\rho}\left(t_{h}\right)\right] \\
& +\sum_{\rho \sigma \alpha} C_{\rho \sigma \alpha}^{i}\left(t_{h}, \omega\right) x_{\pi}^{\alpha}\left(t_{h}, \omega\right)\left[z^{o}\left(t_{h+1}\right)-z^{\rho}\left(t_{h}\right)\right]\left[z^{o}\left(t_{h+1}\right)-z^{\sigma}\left(t_{h}\right)\right]
\end{aligned}
$$

and for $t_{h}<t<t_{h+1}$ we define $x_{\pi}(t, \omega)$ by linear interpolation between $x_{\pi}\left(t_{h}, \omega\right)$ and $x_{\pi}\left(t_{h+1}, \omega\right)$.

Theorem 4 in [5] states hypotheses under which to every $\varepsilon$ there corresponds a $\delta$ such that if mesh $\pi<\delta$, then

$$
\left\|x(t)-x_{\pi}(t)\right\|<\varepsilon, \quad a \leqq t \leqq b .
$$

Unfortunately, we cannot simply apply his theorem, because it only applies to first order integrals, and because although his integrands are extremely general functions, they are nonstochastic. On the 
other hand, the hypotheses for Case 1 of our Theorems 2 and 3, where the Cauchy polygons turn up, are sufficiently strong to make a direct imitation of the proof of his theorem quite straightforward (though there seems to be no way around a singularly gory computation!). Thus the theorem we use is the following:

Cauchy Polygon Theorem. Suppose hypotheses (1)-(4) hold. Suppose $c_{\rho o}^{i}(t, \omega)$ and $C_{\rho \sigma \alpha}^{i}(t, \omega)$ are processes adapted to $\mathscr{F}$ which are bounded on $[a, b]$ uniformly in $\omega$ and which have a.s. continuous sample paths. Suppose $x(t, \omega)$ is a solution to the system of equations

$$
\begin{aligned}
x^{i}(t, \omega)= & y^{i}(\omega)+\int_{a}^{t} \sum_{\rho \alpha} c_{\rho_{\sigma}}^{i}(s, \omega) x^{\alpha}(s, \omega) d z^{\rho} \\
& +\int_{a}^{t} \sum_{\rho \sigma \alpha} C_{\rho \sigma \alpha}^{i}(s, \omega) x^{\alpha}(s, \omega) d z^{\rho} d z^{\sigma},
\end{aligned}
$$

and suppose $x_{\pi}(t, \omega)$ is the Cauchy polygon corresponding to $x(t, \omega)$. Then for every $\varepsilon>0$ there is a $\delta>0$ such that if mesh $\pi<\delta$, then

$$
\left\|x(t, \omega)-x_{\pi}(t, \omega)\right\|<\varepsilon .
$$

\section{Proofs.}

Preliminary Lemma. If $f$ has property $I_{2}$ as defined in the statement of McShane's Theorem 3.1 in Section 1, and if hypotheses (1)-(4) hold, then

$$
\begin{aligned}
& \left\|\int_{0}^{t} f(s) d z^{\rho} d z^{\sigma}\right\| \leqq K_{9}\left[\int_{0}^{t}\|f(s)\|^{2} d s\right]^{1 / 2} \\
& \left\|\int_{0}^{t} f(s) d z^{\rho}\right\| \leqq K_{10}\left[\int_{0}^{t}\|f(s)\|^{2} d s\right]^{1 / 2}
\end{aligned}
$$

where $K_{9}=K_{4}+2 K_{2} T$ and $K_{10}=2 K_{1} T^{1 / 2}+K_{2}^{1 / 2}$.

Proof of Theorem 1. Existence: The proof of existence is an application of the Picard method. Suppose $X_{0}(t, \omega)$ is an $L_{2}$-norm continuous stochastic process with $X_{0}(t, \cdot) \mathscr{F}_{t}$-measurable. If we define $X_{1}(t, \omega)$ as

$$
y^{i}(\omega)+\int_{0}^{t} \sum_{\rho, \alpha} c_{\rho \alpha}^{i}(s, \omega) X_{0}(s, \omega) d z^{\rho}+\int_{0}^{t} \sum_{\rho \sigma \alpha} C_{\rho \sigma \alpha}^{i}(s, \omega) X_{0}(s, \omega) d z^{\rho} d z^{\sigma},
$$

then $X_{1}(t, \omega)$ exists and is $L_{2}$-norm continuous, and $X_{1}(t, \cdot)$ is $\mathscr{F}_{t^{-}}$ measurable.

Proofs of all three of these statements are straightforward applications of standard arguments, assisted by Theorem 3.1 of [4] as stated in $\S 1$ and the Preliminary Lemma. 
Iteration then permits us to define a sequence of processes $X_{n}(t, \omega)$. There remains to be shown that the resulting sequence converges, and that its limit satisfies $(*)$. The latter argument is again standard, but the former is a trifle trickier than usual, so we provide some details:

$$
\begin{aligned}
\left\|X_{2}^{i}(t)-X_{1}^{i}(t)\right\| \leqq & \left\|\int_{0}^{t} \sum_{\alpha \rho} c_{\rho \alpha}^{i}(s)\left[X_{0}^{\alpha}(s)-X_{1}^{\alpha}(s)\right] d z^{\rho}\right\| \\
& +\left\|\int_{0}^{t} \sum_{\alpha \rho \sigma} C_{\rho \sigma \alpha}^{i}(s)\left[X_{1}^{\alpha}(s)-X_{0}^{\alpha}(s)\right] d z^{\rho} d z^{o}\right\| \\
\leqq & K_{10}\left[\int_{0}^{t}\left\|\sum_{\alpha \rho} c_{\rho \alpha}^{i}(s)\left[X_{1}^{\alpha}(s)-X_{0}^{\alpha}(s)\right]\right\|^{2} d s\right]^{1 / 2} \\
& +K_{9}\left[\int_{0}^{t}\left\|\sum_{\rho \sigma \alpha} C_{\rho \sigma \alpha}^{i}(s)\left[X_{1}^{\alpha}(s)-X_{0}^{\alpha}(s)\right]\right\|^{2} d s\right]^{1 / 2} .
\end{aligned}
$$

Let $L$ be the bound on $\left|c_{\rho \alpha}^{i}(s, \omega)\right|$ and $\left|C_{\rho \sigma \alpha}^{i}(s, \omega)\right|$ provided for in the hypotheses. Then

$$
\begin{aligned}
\int_{0}^{t}\left\|\sum_{\rho \alpha} c_{\rho \alpha}^{i}(s)\left[X_{i}^{\alpha}(s)-X_{0}^{\alpha}(s)\right]\right\|^{2} d s & \leqq r L^{2} \int_{0}^{t}\left\|\sum_{\alpha}\left[X_{1}^{\alpha}(s)-X_{0}^{\alpha}(s)\right]\right\|^{2} d s \\
& \leqq r L^{2} \int_{0}^{t}\left\|X_{1}(s)-X_{0}(s)\right\|^{2} d s
\end{aligned}
$$

and

$$
\int_{0}^{t} \sum_{\rho \sigma \alpha} C_{\rho \sigma \alpha}^{i}(s)\left[X_{1}^{\alpha}(s)-X_{0}^{\alpha}(s)\right]\left\|^{2} d s \leqq r^{2} L^{2} \int_{0}^{t}\right\| X_{1}(s)-X_{0}(s) \|^{2} d s,
$$

so if we let $K^{2}=\left[K_{9} r+K_{10} r^{1 / 2}\right] n L$, then

$$
\left\|X_{2}(t)-X_{1}(t)\right\|^{2} \leqq K^{2} \int_{0}^{t}\left\|X_{1}(s)-X_{0}(s)\right\|^{2} d s .
$$

But $X_{0}(t)$ and $X_{1}(t)$ are both $L_{2}$-norm continuous, hence there is a constant $M$ such that $\left\|X_{1}(s)-X_{0}(s)\right\| \leqq M^{2}$ on $[0, T]$, so we have

$$
\left\|X_{2}(t)-X_{1}(t)\right\| \leqq K M t \text {. }
$$

Using the above inequalities, it is simple to prove inductively that for all $n$

$$
\begin{aligned}
& \left\|X_{n}(t)-X_{n-1}(t)\right\|^{2} \leqq M / K[K t]^{n} / n !, \text { i.e., } \\
& \left\|X_{n}(t)-X_{n-1}(t)\right\| \leqq[M / K]^{1 / 2}[K t]^{n / 2} /(n !)^{1 / 2} .
\end{aligned}
$$

But $\sum_{n=1}^{\infty}[K t]^{n / 2} /(n !)^{1 / 2}$ converges uniformly for all $t \in[0, T]$, by a routine power series argument.

Hence by the Weierstrass comparison test, $\sum_{n=1}^{\infty}\left\|X_{n+1}(t)-X_{n}(t)\right\|$ converges uniformly on $[0, T]$. It follows that $\left\|\sum_{n=1}^{\infty}\left[X_{n+1}(t)-X_{n}(t)\right]\right\|<$ $\infty$. But the kth partial sum of $\sum_{n=0}^{\infty}\left[X_{n+1}(t)-X_{n}(t)\right]$ is $X_{b}(t)-X_{0}(t)$. 
So the sequence $X_{0}(t), X_{1}(t), \cdots$ converges uniformly in $t$, for a.a. $\omega$.

Uniqueness. To prove uniqueness we re-apply several of the above computations, this time to $\|X(t)-Y(t)\|$, where $X$ and $Y$ are two solutions of $I$ with the same initial random variable. The induction this time yields that for any $j$

$$
\|X(t)-Y(t)\|^{2} \leqq\left(n^{2} / K\right)\left(\left[K^{2} t\right]^{j} / j !\right) .
$$

Since

$$
\sum_{j}\left[t^{j} / j !\right]<\infty,\|X(t)-Y(t)\|=0 \text { for all } t \in[0, T] .
$$

Before proving Theorems 2 and 3 we need to make some observations about relevant Cauchy polygons. The Cauchy polygons associated with equations I and II are

$$
\begin{aligned}
\left(I_{\pi}\right) \quad x_{\pi}^{i}\left(t_{j+1}\right)= & x_{\pi}^{i}\left(t_{j}\right)+\sum_{h=1}^{n}\left\{\sum_{\rho=1}^{r} A_{h \rho}^{i}\left(t_{j}\right) x_{\pi}^{h}\left(t_{j}\right) \Delta_{j}\left(z^{\rho}\right)\right. \\
& \left.+\sum_{\rho, \sigma=1}^{r} B_{h \rho \sigma}^{i}\left(t_{j}\right) x_{\pi}^{h}\left(t_{j}\right) \Delta_{j} z^{\rho} \Delta_{j} z^{o}\right\} \\
\left(I I_{\pi}\right) \quad y^{i}\left(t_{j+1}\right)= & y^{i}\left(t_{j}\right)+\sum_{h=1}^{n}\left\{-\sum_{\rho=1}^{r} A_{i \rho}^{h}\left(t_{j}\right) y_{\pi}^{h}\left(t_{j}\right) \Delta_{j} z^{\rho}\right. \\
+ & \left.\sum_{\rho, o=1}^{r}\left[-B_{i \rho \sigma}^{h}\left(t_{j}\right)+\sum_{k=1}^{n} A_{k \rho}^{h}\left(t_{j}\right) A_{i \sigma}^{k}\left(t_{j}\right)\right] y_{\pi}^{h}\left(t_{j}\right) \Delta_{j} z^{\rho} \Delta_{j} z^{\sigma}\right\} .
\end{aligned}
$$

For the proofs of both theorems, we need to make the following restrictions on the Cauchy partition $\pi$ being used.

Choose $\varepsilon>0$. We require that $\pi$ be fine enough so that

$$
\begin{aligned}
& \left\|\sum_{i}\left[x^{i}\left(t_{j}\right)-x_{\pi}^{i}\left(t_{2}\right)\right]\right\|<\varepsilon / 3 \text { for all } t_{j} \in \pi \\
& \left\|\sum_{i}\left[y^{i}\left(t_{j}\right)-y_{\pi}^{i}\left(t_{j}\right)\right]\right\|<\varepsilon / 3 \text { for all } t_{j} \in \pi .
\end{aligned}
$$

This restriction on $\pi$ is justified by the Cauchy Polygon Theorem stated at the end of $\S 1$.

(iii) For any $t_{j}, t_{j+1}$ in $\pi$,

$$
E\left(\left[\Delta_{j} z^{\rho}\right]^{c} \mid \mathscr{F}_{t_{j}}\right)<\frac{\varepsilon^{2}\left(t_{j+1}-t_{j}\right)^{2}}{K^{2}[b-a]} \quad c=6,8,
$$

where $K$ is a constant determined from $A_{h \rho}^{i}, B_{h \rho \sigma}^{i}$ in a manner defined in the proof of Theorem 3. This restriction is made possible by hypothesis (5) in the statement of the theorem.

(iv) mesh $\pi \leqq 1$.

With these restrictions available, we are in a position to proceed to the proof of Theorem 2 . 
Case 1. Assume $A_{h \rho}, B_{h \rho_{\sigma}}$ are uniformly bounded on $[a, b]$. Highly temporarily, let

$$
\theta=\sum_{h \rho} A_{h \rho}^{i}\left(t_{j}\right) x_{\pi}^{h}\left(t_{j}\right) \Delta_{j} z^{\rho}+\sum_{h \rho \sigma} B_{h \rho \sigma}^{i}\left(t_{j}\right) x_{\pi}^{h}\left(t_{j}\right) \Delta_{j} z^{\rho} \Delta_{j} z^{\sigma} .
$$

Then $I_{\pi}$ gives us that

$$
\begin{aligned}
{\left[x_{\pi}^{i}\left(t_{j+1}\right)\right]^{4}=} & {\left[x_{\pi}^{i}\left(t_{j}\right)\right]^{4}+4\left[x_{\pi}^{i}\left(t_{j}\right)\right]^{3} \theta+6\left[x_{\pi}^{i}\left(t_{j}\right)\right]^{2} \theta^{2}+4 x_{\pi}^{i}\left(t_{j}\right) \theta^{3}+\theta^{4} } \\
= & {\left[x_{\pi}^{i}\left(t_{j}\right)\right]^{4}+4\left[x_{\pi}^{i}\left(t_{j}\right)\right]^{3} \sum_{h \rho} A_{h \rho}^{i}\left(t_{j}\right) x_{\pi}^{h}\left(t_{j}\right) \Delta_{j} z^{\rho} } \\
& +4\left[x_{\pi}^{i}\left(t_{j}\right)\right]^{3} \sum_{h \rho \sigma} B_{h \rho \sigma}^{i}\left(t_{j}\right) x_{\pi}^{h}\left(t_{j}\right) \Delta_{j} z^{\rho} \Delta_{j} z^{\sigma} \\
& +6\left[x_{\pi}^{i}\left(t_{j}\right)\right]^{2} \sum_{h \rho_{\rho} \sigma} A_{h \rho}^{i}\left(t_{j}\right) A_{k \sigma}^{i}\left(t_{j}\right) x_{\pi}^{h}\left(t_{j}\right) x_{\pi}^{k}\left(t_{j}\right) \Delta_{j} z^{\rho} \Delta_{j} z^{\sigma} \\
& + \text { multitudinous terms, each containing from } 3 \text { to } 8
\end{aligned}
$$

$\Delta_{j} z^{\rho}$ factors, $4 x_{\pi}^{i}$ factors and various $A_{h \rho}^{i}$ and $B_{h \rho \sigma}^{i}$ factors. Now we let

$$
M=\max _{i, \rho, a, h, \omega}\left(1, \sup _{t \in[a, b]}\left|A_{h \rho}^{i}(t)\right|, \sup _{t \in[a, b]}\left|B_{h \rho \sigma}^{i}(t)\right|\right)
$$

and

$$
v\left(t_{j}\right)=\max _{i} E\left(\left[x_{\pi}^{i}\left(t_{j}\right)\right]^{4}\right) .
$$

What we are going to do is to show that there exists a number $\hat{K}$ such that

$$
\begin{gathered}
v\left(t_{j+1}\right)-v\left(t_{j}\right) \leqq \hat{K} v\left(t_{j}\right)\left(t_{j+1}-t_{j}\right), \quad \text { i.e., } \\
v\left(t_{j+1}\right) \leqq v\left(t_{0}\right)+\hat{K} \sum_{i=0}^{j} v\left(t_{i}\right)\left(t_{i+1}-t_{i}\right) .
\end{gathered}
$$

Then we shall define a function $\widetilde{v}$ on the whole interval $[a, b]$ which is equal to $v$ at $t=t_{0}, \cdots, t_{m}$, and apply Gronwall's Lemma to $\widetilde{v}(t)$. From the result of the application we will be able to deduce that

$$
E\left(\left(x_{\pi}^{i}\left(t_{j}\right)\right)^{4}\right) \leqq \frac{v\left(t_{0}\right)}{e^{\hat{K} t_{0}}} e^{\hat{K} b} \text { for all } t_{j} \text { in } \pi \text { and } i=1, \cdots, n .
$$

We have

$$
E\left[\left(x_{\pi}^{i}\left(t_{j}\right)\right)^{4}\right] \leqq v\left(t_{j}\right)
$$

and

$$
E\left(\sum_{\rho h}\left(x_{\pi}^{i}\left(t_{j}\right)\right)^{3}\left(x_{\pi}^{h}\left(t_{j}\right)\right) A_{h \rho}^{i}\left(t_{j}\right) \Delta_{j} z^{\rho}\right) \leqq M E\left(\sum_{h}\left(x_{\pi}^{i}\left(t_{j}\right)\right)^{3}\left(x_{\pi}^{h}\left(t_{j}\right)\right) \sum_{\rho} \Delta_{j} z^{\rho}\right) .
$$

Now for each $h$ we have 


$$
\left(x_{\pi}^{i}(t)\right)^{3} x_{\pi}^{h}\left(t_{j}\right) \leqq \max _{i}\left(x_{\pi}^{i}\left(t_{j}\right)\right)^{4} \leqq \sum_{i}\left(x_{\pi}^{i}\left(t_{j}\right)\right)^{4} .
$$

It follows that

$$
\sum_{h}\left(x_{\pi}^{i}\left(t_{j}\right)\right)^{3} x_{\pi}^{h}\left(t_{j}\right) \leqq n \sum_{i}\left(x_{\pi}^{i}\left(t_{j}\right)\right)^{4}
$$

Using this we have

$$
\begin{aligned}
E\left(\sum_{h}\left(x_{\pi}^{i}\left(t_{j}\right)\right)^{3} x_{\pi}^{h}\left(t_{j}\right) \sum_{\rho} \Delta_{j} z^{\rho}\right)= & E\left\{\sum_{h}\left(x_{\pi}^{i}\left(t_{j}\right)\right)^{3} x_{\pi}^{h}\left(t_{j}\right) E\left(\sum_{\rho} \Delta_{j} z^{\rho} \mid \mathscr{F}_{t_{j}}\right)\right\} \\
& \leqq n \sum_{i} E\left(\left(x_{\pi}^{i}\left(t_{j}\right)\right)^{4}\right) r K_{1}\left(t_{j+1}-t_{j}\right)[\text { hyp. 4] } \\
& \leqq n^{2} v\left(t_{j}\right) r K_{1}\left(t_{j+1}-t_{j}\right)
\end{aligned}
$$

i.e.,

$$
4 E\left\{\left[x_{\pi}^{i}\left(t_{j}\right)\right]^{3} \sum_{h \rho} A_{h \rho}^{i}\left(t_{j}\right) x_{\pi}^{h}\left(t_{j}\right) \Delta_{j} z^{\rho}\right\} \leqq 4 M n^{2} v\left(t_{j}\right) r K_{1}\left(t_{j+1}-t_{j}\right) .
$$

Similarly,

$$
4 E\left\{\left[x_{\pi}^{i}\left(t_{j}\right)\right]^{3} \sum_{h \rho \sigma} B_{h \rho \sigma}^{i}\left(t_{j}\right) x_{\pi}^{h}\left(t_{j}\right) \Delta_{j} z^{\rho} \Delta_{j} z^{\sigma}\right\} \leqq 4 M n^{2} r^{2} K_{2}\left(t_{j+1}-t_{j}\right) v\left(t_{j}\right) .
$$

Likewise

$$
\begin{aligned}
& 6 E\left(x_{\pi}^{i}\left(t_{j}\right)\right)^{2} \sum_{h k \rho \sigma} x_{\pi}^{h}\left(t_{j}\right) x_{\pi}^{k}\left(t_{j}\right) A_{h \rho}\left(t_{j}\right) A_{k \sigma}^{i}\left(t_{j}\right)\left(\Delta_{j} z^{\rho} \Delta_{j} z^{o}\right) \\
& \quad \leqq 6 M^{2} n^{3} v\left(t_{j}\right) r^{2} K_{2}\left(t_{j+1}-t_{j}\right) .
\end{aligned}
$$

Before tackling the remaining herd of terms we need to make a few observations:

(a) By hypothesis (iii) in the choice of $\pi$

$$
\frac{E\left(\left[z^{\rho}\left(t_{j+1}\right)-z^{\rho}\left(t_{j}\right)\right]^{c} \mid \mathscr{F}_{t_{j}}\right)}{\left(t_{j+1}-t_{j}\right)^{2}} \leqq \frac{\varepsilon^{2}}{K^{2}[b-a]} \text { a.e. } c=6,8 .
$$

Hence, using hypothesis (iv) in the choice of $\pi$,

$$
\frac{E\left(\left[z^{\rho}\left(t_{j+1}\right)-z^{o}\left(t_{j}\right)\right]^{c} \mid \mathscr{F}_{t_{j}}\right)}{\left(t_{j+1}-t_{j}\right)} \leqq \frac{\varepsilon^{2}}{K^{2}[b-a]} \quad \text { a.e. } \quad c=6,8 .
$$

Let

$$
B=\max \left\{K_{2}, K_{4}, \frac{\varepsilon^{2}}{K^{2}[b-a]}\right\} .
$$

(b) From (a) and hypothesis 4 it follows that

$$
\frac{E\left(\left[z^{\rho}\left(t_{j+1}\right)-z^{\rho}\left(t_{j}\right)\right]^{c} \mid \mathscr{F}_{t_{j}}\right)}{t_{j+1}-t_{j}} \leqq B \text { for } c=3, \cdots, 8 .
$$


(c) In any term of the expansion of $\left[x_{\pi}^{i}\left(t_{j+1}\right)\right]^{4}$ there are at most 4 factors of the form $A_{h \rho}^{i}\left(t_{j}\right)$ or $B_{h \rho \sigma}^{i}\left(t_{j}\right)$. Hence the contribution from such factors must be less than or equal to $M^{4}$.

So each of the remaining terms of the expansion must be less than or equal to

$$
M^{4} E\left(\sum_{h k l m}\left|x_{\pi}^{h}\left(t_{j}\right) x_{\pi}^{k}\left(t_{j}\right) x_{\pi}^{l}\left(t_{j}\right) x_{\pi}^{m}\left(t_{j}\right) \sum_{\sigma_{1}, \cdots, \sigma_{q}} \Delta_{j} z^{\sigma_{1}}, \cdots, \Delta_{j} z^{\sigma_{q}}\right|\right)
$$

(where $q \in\{3, \cdots, 8\}$ )

$$
\begin{aligned}
& \leqq M^{4} n^{3} E\left\{\sum_{i}\left(x_{\pi}^{i}\right)^{4} E\left(\sum_{\sigma_{1}, \cdots, \sigma_{q}}\left|\Delta_{j} z^{\sigma_{1}}, \cdots, \Delta_{j} z^{\sigma_{q}}\right| \mid \mathscr{F}_{t_{j}}\right)\right\} \\
& \leqq M^{4} n^{3} r^{8} B v\left(t_{j}\right)\left(t_{j+1}-t_{j}\right) .
\end{aligned}
$$

Counting coefficients, there are 66 such terms, so the total conglomeration of terms involving 3 or more $\Delta_{j} z^{\sigma}$ 's is

$$
\leqq 66 M^{4} n^{3} r^{8} B v\left(t_{j}\right)\left(t_{j+1}-t_{j}\right)
$$

Collecting all the information on the past several pages, we have that if $\hat{K}=4 n^{2} r K_{1} M+4 M n^{2} r^{2} K_{2}+6 M^{2} n^{3} r^{2} K_{2}+66 M^{4} n^{3} r^{8} B$, then $v\left(t_{j+1}\right)-v\left(t_{j}\right) \leqq \hat{K} v\left(t_{j}\right)\left(t_{j+1}-t_{j}\right)$.

Summing up to $j+1$ this gives

$$
v\left(t_{j+1}\right) \leqq v\left(t_{0}\right)+\hat{K} \sum_{i=0}^{j} v\left(t_{i}\right)\left(t_{i+1}-t_{i}\right) .
$$

We define a function $\widetilde{v}(t)$ on $[a, b]$ by $\widetilde{v}(t)=v\left(t_{j}\right)$, where $t_{j}$ is the largest partition point of $\pi$ which is $\leqq t$.

From (**), we have that

$$
\widetilde{v}(t) \leqq \widetilde{v}\left(t_{0}\right)+\hat{K} \int_{t_{0}}^{t} \widetilde{v}(s) d s
$$

By Gronwall's Lemma, if we can find a function satisfying

$$
g(t)=\widetilde{v}\left(t_{0}\right)+\hat{K} \int_{t_{0}}^{t} g(s) d s,
$$

then we will have that

$$
\widetilde{v}(t) \leqq g(t) \text { for all } t \in[a, b] .
$$

Let

$$
g(t)=\frac{\widetilde{v}\left(t_{0}\right)}{e^{\hat{K} t_{0}}} e^{\hat{K} t}
$$

Then 


$$
\left.\hat{K} \int_{t_{0}}^{t} g(s) d s=\hat{K} \frac{\widetilde{v}\left(t_{0}\right)}{e^{\hat{K} t_{0}}} \cdot \frac{1}{\hat{K}} e^{\hat{K} s}\right]_{t_{0}}^{t}=g(t)-\widetilde{v}\left(t_{0}\right) .
$$

Hence

$$
v\left(t_{j}\right) \leqq \frac{v\left(t_{0}\right)}{e^{\hat{K} t_{0}}} e^{\hat{K} t_{j}} \text { for all } j
$$

Since $e^{t}$ increases with $t$, this gives us that

$$
E\left\{\left[x_{\pi}^{i}\left(t_{j}\right)\right]^{4}\right\} \leqq \frac{v\left(t_{0}\right)}{e^{\hat{K} t_{0}}} e^{\hat{K} b} \text { for all } t_{j} \in \pi \text { and all } i .
$$

Since $\left[x_{\pi}^{i}\right]^{4}$ is an increasing function, and since $x_{\pi}^{i}$ approximates $x^{i}$, this completes the proof of Case 1 .

Case 2. The removal of the uniform boundedness of $A_{h \rho}^{i}$ and $B_{h \rho \sigma}^{i}$ uses an argument which is a simplification of the argument used to perform the same feat in Theorem 3; we will therefore omit it.

Proof of Corollary. Only one observation is needed in order to make the proof of the corollary an exact imitation of the proof of Theorem 2. That is the observation that the hypothesis that

$$
\lim \operatorname{ess} \sup \frac{E\left(\left[z^{\rho}(t)-z^{\rho}(s)\right]^{\circ} \mid \mathscr{F}_{s}\right)}{(t-s)^{2}}=0 \quad \text { uniformly }
$$

on $[a, b]$ for $c=6$ and $c=2 q$ implies the same hypothesis for any $n$ with $6 \leqq n \leqq 2 q$. This we get by applying Hölder's inequality to the product $\left|z^{\rho}(t)-z^{\rho}(s)\right|^{6 h}\left|z^{\rho}(t)-z^{\rho}(s)\right|^{2 q k}$, where $h=(2 q-n) /(2 q-6)$ and $k=(n-6) /(2 q-6)$.

Proof of Theorem 3. Remark. Since $x^{i}(\alpha), y^{i}(\alpha)$ are bounded a.e., by eliminating a set of measure zero we may consider them to be bounded for all $\omega$.

Second Remark. We have sufficient hypotheses to guarantee the existence of a solution to II (using Theorem 1). It will, therefore, suffice to show that this solution has the desired properties.

Case 1. Add on the hypothesis that $A_{h \rho}^{i}(t, \omega), B_{h \rho \sigma}^{i}(t, \omega)$ be uniformly bounded on $[a, b]$ (i.e., bounded uniformly in $\omega$ ). The first part of the proof is a horrendous computation with Cauchy polygons. What we want to consider is

$$
\left\|\sum_{i=1}^{n} x_{\pi}^{i}\left(t_{j+1}\right) y_{\pi}^{i}\left(t_{j+1}\right)-x_{\pi}^{i}\left(t_{j}\right) y_{\pi}^{i}\left(t_{j}\right)\right\| .
$$




$$
\begin{aligned}
x_{\pi}^{i}\left(t_{j+1}\right) y_{\pi}^{i}\left(t_{j+1}\right)-x_{\pi}^{i}\left(t_{j}\right) y_{\pi}^{i}\left(t_{j}\right)= & {\left[x_{\pi}^{i}\left(t_{j+1}\right)-x_{\pi}^{i}\left(t_{j}\right)\right] y_{\pi}^{i}\left(t_{j+1}\right) } \\
& +x_{\pi}^{i}\left(t_{j}\right)\left[y_{\pi}^{i}\left(t_{j+1}\right)-y_{\pi}^{i}\left(t_{j}\right)\right] .
\end{aligned}
$$

We use $I_{\pi}$ and $I I_{\pi}$ to replace $x_{\pi}^{i}\left(t_{j+1}\right)$ and $y_{\pi}^{i}\left(t_{j+1}\right)$ and find that the above yields

(3)

$$
\begin{aligned}
& \sum_{\rho=1}^{r} \Delta_{j} z^{\rho}\left\{\sum_{h=1}^{n} A_{h \rho}^{i}\left(t_{j}\right) x_{\pi}^{h}\left(t_{j}\right) y_{\pi}^{i}\left(t_{j}\right)-\sum_{h=1}^{n} A_{i \rho}^{h}\left(t_{j}\right) x_{\pi}^{i}\left(t_{j}\right) y_{\pi}^{h}\left(t_{j}\right)\right\} \\
& \quad+\sum_{\rho, \sigma=1}^{r} \Delta_{j} z^{\rho} \Delta_{j} z^{\sigma}\left\{\sum_{h=1}^{n} B_{h \rho o}\left(t_{j}\right) x_{\pi}^{h}\left(t_{j}\right) y_{\pi}^{i}\left(t_{j}\right)\right. \\
& \quad-\sum_{h, k=1}^{n} A_{h \rho}^{i}\left(t_{j}\right) A_{i o}^{k}\left(t_{j}\right) x_{\pi}^{h}\left(t_{j}\right) y_{\pi}^{i}\left(t_{j}\right) \\
& \left.\quad-\sum_{h=1}^{r} B_{i \rho \sigma}^{h}\left(t_{j}\right) x_{\pi}^{i}\left(t_{j}\right) y_{\pi}^{h}\left(t_{j}\right)+\sum_{h, k=1}^{n} A_{k o}^{h}\left(t_{j}\right) A_{i \rho}^{k}\left(t_{j}\right) x_{\pi}^{2}\left(t_{j}\right) y_{\pi}^{h}\left(t_{j}\right)\right\} \\
& \quad+\sum_{\rho, \sigma, \tau=1}^{r} \Delta_{j} z^{o} \Delta_{j} z^{\sigma} \Delta_{j} z^{\tau}\left\{-\sum_{h, k=1}^{n} B_{h \rho o}^{i}\left(t_{j}\right) x_{\pi}^{h}\left(t_{j}\right) A_{i \tau}^{l}\left(t_{j}\right) y_{\pi}^{l}\left(t_{j}\right)\right. \\
& \left.\quad+\sum_{h, l=1}^{n} A_{h \rho}^{i}\left(t_{j}\right)\left[-B_{i \sigma \tau}^{l}\left(t_{j}\right)+\sum_{k=1}^{n} A_{k \tau}^{l}\left(t_{j}\right) A_{i \sigma}^{k}\left(t_{j}\right)\right] x_{\pi}^{h}\left(t_{j}\right) y_{\pi}^{l}\left(t_{j}\right)\right\} \\
& \quad+\sum_{\rho, \sigma, \tau, \nu=1}^{r} \Delta_{j} z^{\rho} \Delta_{j} z^{\sigma} \Delta_{j} z^{\tau} \Delta_{j} z^{\nu}\left\{\sum _ { h , l = 1 } ^ { n } B _ { h \rho \sigma } ^ { i } ( t _ { j } ) \left[-B_{i \tau \nu}^{l}\left(t_{j}\right)\right.\right. \\
& \left.\left.\quad+\sum_{k=1}^{n} A_{k \nu}^{l}\left(t_{j}\right) A_{i \tau}^{k}\left(t_{j}\right)\right]\left[x_{\pi}^{h}\left(t_{j}\right) y_{\pi}^{l}\left(t_{j}\right)\right]\right\} .
\end{aligned}
$$

Consider this term by term:

(1) Since we are summing also over $i=1, \cdots, n$ we can re-label various terms:

$$
\sum_{i=1}^{n}(1)=\sum_{\rho=1}^{n} \Delta_{j} z^{\rho}\left\{\sum_{h, i=1}^{n} A_{h \rho}^{i}\left(t_{j}\right) x_{\pi}^{h}\left(t_{j}\right) y_{\pi}^{i}\left(t_{j}\right)-\sum_{h, i=1}^{n} A_{h \rho}^{i}\left(t_{j}\right) x_{\pi}^{h}\left(t_{j}\right) y_{\pi}^{i}\left(t_{j}\right)\right\}=0 .
$$

Likewise for (2):

$$
\begin{aligned}
\sum_{i=1}^{n}(2)= & \sum_{\rho, \sigma=1}^{r} \Delta_{j} z^{\rho} \Delta_{j} z^{\sigma}\left\{\sum_{h, i=1}^{n} B_{h \rho \sigma}^{i}\left(t_{j}\right) x_{\pi}^{h}\left(t_{j}\right) y_{\pi}^{i}\left(t_{j}\right)\right. \\
& -\sum_{h, i=1}^{n} B_{h \rho \sigma}^{i}\left(t_{j}\right) x_{\pi}^{h}\left(t_{j}\right) y_{\pi}^{i}\left(t_{j}\right)-\sum_{h, k, i=1}^{n} A_{h \rho}^{i}\left(t_{j}\right) A_{i o}^{k}\left(t_{j}\right) x_{\pi}^{h}\left(t_{j}\right) y_{\pi}^{k}\left(t_{j}\right) \\
& \left.+\sum_{h, k, i=1}^{n} A_{i o}^{k}\left(t_{j}\right) A_{h \rho}^{i}\left(t_{j}\right) x_{\pi}^{h}\left(t_{j}\right) y_{\pi}^{k}\left(t_{j}\right)\right\}=0 .
\end{aligned}
$$

So what is needed to complete the proof is that $\left\|\sum_{i=1}^{n}(3)+(4)\right\|$ can be made arbitrarily small.

To prevent writer's cramp we define $K_{h k \rho \sigma \tau}^{i}\left(t_{j}\right)$ and $\widetilde{K}_{h k \rho \sigma \varepsilon \nu}^{i}\left(t_{j}\right)$ by

$$
\begin{gathered}
K_{h l \rho \sigma \tau}^{i}\left(t_{j}\right)=-B_{h \rho \sigma}^{i}\left(t_{j}\right) A_{i \tau}^{l}\left(t_{j}\right)+A_{h \rho}^{i}\left(t_{j}\right)\left[-B_{i \sigma \tau}^{l}\left(t_{j}\right)+\sum_{k=1}^{n} A_{k \tau}^{l}\left(t_{j}\right) A_{i \sigma}^{k}\left(t_{j}\right)\right] \\
\widetilde{K}_{h l \rho \sigma \tau \nu}^{i}\left(t_{j}\right)=B_{h \rho \sigma}^{i}\left(t_{j}\right)\left[-B_{i \tau \nu}^{l}\left(t_{j}\right)+\sum_{k=1}^{n} A_{k \nu}^{l}\left(t_{j}\right) A_{i \tau}^{k}\left(t_{j}\right)\right] .
\end{gathered}
$$


Since we assumed $A_{h \rho}^{i}$ and $B_{h \rho \sigma}^{i}$ to be uniformly bounded on $[a, b] \times \Omega$ for $i, h=1, \cdots, n ; \rho, \sigma=1, \cdots, r$, it follows that $K_{h l \rho \sigma \tau}^{i}(t)$ and $\widetilde{K}_{h l \rho \sigma \tau \nu}^{i}(t)$ must also be uniformly bounded on $[a, b] \times \Omega$. Say

$$
\left.\begin{array}{c}
K_{h l o \rho \tau}^{i}(t) \leqq K \\
\widetilde{K}_{h l o \rho=\nu}^{i}(t) \leqq \widetilde{K}
\end{array}\right\} \quad \text { on } \quad[a, b] \times \Omega
$$

We fix $\rho, \sigma, \tau$, and $i$ and consider summands of $\|$ (3) $\|$.

$$
\begin{aligned}
& \left\|\sum_{h, k=1}^{n} \Delta_{j} z^{\rho} \Delta_{j} z^{\sigma} \Delta_{j} z^{\tau} K_{h l o \rho \tau}^{i}\left(t_{j}\right) x_{\pi}^{h}\left(t_{j}\right) y_{\pi}^{k}\left(t_{j}\right)\right\| \\
& \quad \leqq\left\|\sum_{h, k=1}^{n} \Delta_{j} z^{\rho} \Delta_{j} z^{\sigma} \Delta_{j} z^{\tau} x_{\pi}^{h}\left(t_{j}\right) y_{\pi}^{k}\left(t_{j}\right) K\right\| \\
& \quad=K\left\{E\left[\Delta_{j} z^{o} \Delta_{j} z^{\sigma} \Delta_{j} z^{\tau} \sum_{h, k} x_{\pi}^{h}\left(t_{j}\right) y_{\pi}^{k}\left(t_{j}\right)\right]^{2}\right\}^{1 / 2} \\
& \quad=K\left\{E\left(E\left(\left(\Delta_{j} z^{\rho} \Delta_{j} z^{\sigma} \Delta_{j} z^{\tau}\right)^{2}\left(\sum_{h, k} x_{\pi}^{h}\left(t_{j}\right) y_{\pi}^{k}\left(t_{j}\right)\right)^{2} \mid \mathscr{F}_{t_{j}}\right)\right)\right\}^{1 / 2} \\
& \left.\quad=K\left\{E\left(\left(\sum_{h, k} x_{\pi}^{h}\left(t_{j}\right) y_{\pi}^{k}\left(t_{j}\right)\right)^{2}\right) E\left(\left(\Delta_{j} z^{\rho} \Delta_{j} z^{\sigma} \Delta_{j} z^{\tau}\right)^{2} \mid \mathscr{F}_{t_{j}}\right)\right)\right\}^{1 / 2}
\end{aligned}
$$

because $\left(\sum_{h, k} x_{\pi}^{h}\left(t_{j}\right) y_{\pi}^{k}\left(t_{j}\right)\right)^{2}$ is bounded and summable w.r.t $\mathscr{F}_{t_{j}}$. Now $\left(\Delta_{j} z^{\rho} \Delta_{j} z^{\rho} \Delta_{j} z^{\tau}\right)^{2} \leqq\left(\max _{\nu=\rho_{\sigma \tau}} \Delta_{j} z^{\nu}\right)^{6}$. For simplicity we assume the largest is $\Delta_{j} z^{\rho}$.

By hypothesis (iii) in the choice of $\pi$, if $t_{j}, t_{j+1} \in \pi$,

$$
E\left(\left[\Delta_{j} z^{p}\right]^{6} \mid \mathscr{F}_{t_{j}}\right)<\frac{\varepsilon^{2}\left(t_{j+1}-t_{j}\right)^{2}}{K^{2}[b-a]} .
$$

Then we have for $t_{j}, t_{j+1} \in \pi$,

$$
\begin{aligned}
& \left\|\sum_{h, k} \Delta_{j} z^{\rho} \Delta_{j} z^{\sigma} \Delta_{j} z^{\tau} x_{\pi}^{h}\left(t_{j}\right) y_{\pi}^{k}\left(t_{j}\right) K_{h k o \rho_{\tau}}\left(t_{j}\right)\right\| \\
& \leqq K\left\{\left(\left(E\left[\Delta_{j} z^{\rho}\right]^{6} \mid \mathscr{F}_{t_{j}}\right)\right) E\left(\sum_{h, k} x_{\pi}^{h}\left(t_{j}\right) y_{\pi}^{k}\left(t_{j}\right)\right)^{2}\right\}^{1 / 2} \\
& \leqq \frac{\varepsilon\left[t_{j+1}-t_{j}\right]}{[b-a]}\left\{E\left(\sum_{h, k} x_{\pi}^{h}\left(t_{j}\right) y_{\pi}^{k}\left(t_{j}\right)\right)^{2}\right\}^{1 / 2} .
\end{aligned}
$$

So what we need is a bound on all terms

$$
E\left(\sum_{h, k, l, m} x_{\pi}^{h}\left(t_{j}\right) x_{\pi}^{l}\left(t_{j}\right) y_{\pi}^{k}\left(t_{j}\right) y_{\pi}^{m}\left(t_{j}\right)\right)
$$

But

$$
x_{\pi}^{h}\left(t_{j}\right) x_{\pi}^{l}\left(t_{j}\right) y_{\pi}^{h}\left(t_{j}\right) y_{\pi}^{m}\left(t_{j}\right) \leqq\left(\max _{h, k}\left(x_{\pi}^{h}\left(t_{j}\right) y_{\pi}^{k}\left(t_{j}\right)\right)\right)^{4},
$$

and Theorem 2 gives us a bound for $E\left\{\left(x_{\pi}^{h}\left(t_{j}\right)\right)^{4}\right\}$ and $E\left\{\left(y_{\pi}^{k}\left(t_{j}\right)\right)^{4}\right\}$, so we have the desired bound-call it $\widetilde{B}^{2}$. 
Plugging this into the above inequalities we see that

$$
\left\|\sum_{h, k} K_{h k \rho \sigma \tau}\left(t_{j}\right) \Delta_{j} z^{\rho} \Delta_{j} z^{\sigma} \Delta_{j} z^{\tau} x_{\pi}^{h}\left(t_{j}\right) y_{\pi}^{k}\left(t_{j}\right)\right\| \leqq \frac{\varepsilon \widetilde{B}\left(t_{j+1}-t_{j}\right)}{[b-a]} .
$$

Hence even if $\|$ (3) $\|$ varies as much as it can on each interval of $\pi$, the total amount it varies on $[a, b]$ is less than $r^{3} \varepsilon \widetilde{B}$.

By the same argument, the total amount \|(4) $\|$ can vary over $[a, b]$ is less than $r^{4} \widetilde{B} \varepsilon$.

It follows that

$$
\left\|x_{\pi}^{i}\left(t_{j+1}\right) y_{\pi}^{i}\left(t_{j+1}\right)-x_{\pi}^{i}(a) y_{\pi}^{i}(a)\right\|<2 \varepsilon r^{4} B,
$$

hence that

$$
\left\|x_{\pi}\left(t_{j+1}\right) y_{\pi}\left(t_{j+1}\right)-x_{\pi}(a) y_{\pi}(a)\right\|<2 \varepsilon r^{4} \widetilde{B} n
$$

Now

$$
\begin{aligned}
& \left\|x\left(t_{j+1}\right) y\left(t_{j+1}\right)-x(a) y(a)\right\| \leqq\left\|x\left(t_{j+1}\right) y\left(t_{j+1}\right)-x_{\pi}\left(t_{j+1}\right) y_{\pi}\left(t_{j+1}\right)\right\| \\
& \quad+\left\|x_{\pi}\left(t_{j+1}\right) y_{\pi}\left(t_{j+1}\right)-x_{\pi}(a) y_{\pi}(a)\right\|+\left\|x_{\pi}(a) y_{\pi}(a)-x(a) y(a)\right\| \\
& \leqq \\
& \quad+\left\|x\left(t_{j+1}\right)\left[y\left(t_{j+1}\right)-y_{\pi}\left(t_{j+1}\right)\right]\right\| \\
& \quad+\left\|\left[x\left(t_{j+1}\right)-x_{\pi}\left(t_{j+1}\right)\right] y_{\pi}\left(t_{j+1}\right)\right\|+2 \varepsilon r^{4} \widetilde{B} n+0 .
\end{aligned}
$$

Since all hypotheses for Theorem 1 are satisfied, $x(t, \omega)$ and $y(t, \omega)$ could be arrived at by a Picard type process. It follows that $\|x(t)\|$ and $\left\|y_{\pi}(t)\right\|$ are uniformly bounded; say $\left.\|x(t)\|\right\}, \hat{B}$ for all $t$. By our choice of $\pi$, we then have

$$
\left\|x\left(t_{j+1}\right) y\left(t_{j+1}\right)-x(a) y(a)\right\| \leqq 2 \widehat{B} \varepsilon+2 \varepsilon r^{4} \widetilde{B} n=\left(2 \hat{B}+2 r^{4} \widetilde{B} n\right) \varepsilon .
$$

Since any point of $[a, b]$ can be a division point of a Cauchy partition, we therefore have that

$$
x(t) y(t)=x(a) y(a) \quad \text { a.s. }
$$

Case 2. We now remove the spare hypothesis of uniform boundedness and assume merely that $A_{h \rho}^{i}(t, \omega)$ and $B_{h \rho \sigma}^{i}(t, \omega)$ are bounded on $[a, b]$ for a.a. $\omega$. For simplicity we shall drop the subscripts on $A$ and $B$, since they are now irrelevant. Define

$$
\begin{aligned}
& A_{N}^{i}(t, \omega) \text { to be }\left\{\begin{array}{l}
A^{i}(t, \omega) \text { if } A^{i}(s, \omega) \leqq N \text { for all } s \leqq t \\
0 \text { if not; }
\end{array}\right. \\
& B_{N}^{i}(t, \omega) \text { to be }\left\{\begin{array}{l}
B^{i}(t, \omega) \text { if } B^{i}(s, \omega) \leqq N \text { for all } s \leqq t \\
0 \text { if not ; }
\end{array}\right.
\end{aligned}
$$

$x_{N}^{i}(t, \omega), y_{N}^{i}(t, \omega)$ to be the solutions to I and II obtained by substituting $A_{N}^{i}$ and $B_{N}^{i}$ for $A^{i}$ and $B^{i}$. 
Using the metric of convergence in probability defined by

$$
d_{P}(u, v)=\inf \{\delta: P(|u-v| \geqq \delta)<\delta\}
$$

we shall show that for each $t$,

$$
\begin{aligned}
& d_{P}\left(\sum_{i}\left[x^{i}(t, \omega) y^{i}(t, \omega)-x^{i}(a, \omega) y^{i}(a, \omega)\right]\right)=0 . \\
& d_{P}\left(\sum_{i}\left[x^{i}(t, \omega) y^{i}(t, \omega)-x^{i}(a, \omega) y^{i}(a, \omega)\right]\right) \\
& \leqq \\
& d_{P}\left(\sum_{i}\left[x^{i}(t, \omega) y^{i}(t, \omega)-x_{N}^{i}(t, \omega) y_{N}^{i}(t, \omega)\right]\right) \\
& \quad+d_{P}\left(\sum_{i}\left[x_{N}^{i}(t, \omega) y_{N}^{i}(t, \omega)-x_{N}^{i}(a, \omega) y_{N}^{i}(a, \omega)\right]\right) \\
& \quad+d_{P}\left(\sum_{i}\left[x_{N}^{i}(a, \omega) y_{N}^{i}(a, \omega)-x^{i}(a, \omega) y^{i}(a, \omega)\right]\right) .
\end{aligned}
$$

By our opening remark, $x^{i}(a, \omega)$ and $y^{i}(a, \omega)$ are bounded, hence if $N$ is large enough, the third term is zero.

By Case 1, the second term is zero.

That leaves the first term.

Fix $\varepsilon>0$. Since $A^{i}$ and $B^{i}$ are bounded on $[a, b]$ for a.a. $\omega$, there exist $N_{A^{i}}, N_{B^{i}}$ such that

$$
\begin{aligned}
& P\left\{A_{N_{A} i}^{i}(t, \omega) \neq A^{i}(t, \omega)\right\}<\varepsilon / 2 \\
& P\left\{B_{N_{B} i}^{i}(t, \omega) \neq B^{i}(t, \omega)\right\}<\varepsilon / 2 .
\end{aligned}
$$

Let

$$
N=\max _{i=1, \cdots, n}\left\{N_{A^{i}}, N_{B^{i}}\right\}
$$

Then

$$
P\left\{A_{N}^{i}(t, \omega) \neq A^{i}(t, \omega) \quad \text { or } \quad B_{N}^{i}(t, \omega) \neq B^{i}(t, \omega)\right\}<\varepsilon / 2 .
$$

Hence

$$
P\left\{x_{N}^{i}(t, \omega) \neq x^{i}(t, \omega) \quad \text { or } \quad y_{N}^{i}(t, \omega) \neq y^{i}(t, \omega)\right\}<\varepsilon / 2 .
$$

So

$$
P\left\{\sum_{i}\left|x^{i}(t, \omega) y^{i}(t, \omega)-x_{N}^{i}(t, \omega) y_{N}^{i}(t, \omega)\right| \geqq \varepsilon / 2\right\}<\varepsilon / 2 .
$$

Hence

$$
d_{P}\left(\sum_{i}\left[x^{i}(t, \omega) y^{i}(t, \omega)-x_{N}^{i}(t, \omega) y_{N}^{i}(t, \omega)\right]\right) \leqq \varepsilon / 2<\varepsilon .
$$

Since $\varepsilon$ was arbitrary, 


$$
d_{P}\left(\sum_{i}\left[x^{i}(t, \omega) y^{i}(t, \omega)-x_{N}^{i}(t, \omega) y_{N}^{i}(t, \omega)\right]\right)=0
$$

So

$$
d_{P}\left(\sum_{i}\left[x^{i}(t, \omega) y^{i}(t, \omega)-x^{i}(a, \omega) y^{i}(a, \omega)\right]\right)=0 .
$$

Since $x^{i}$ and $y^{i}$ are a.s. continuous, it follows that

$$
\sum_{i} x^{i}(t, \omega) y^{i}(t, \omega)=\sum_{i} x^{i}(a, \omega) y^{i}(\alpha, \omega) \quad \text { a.s. }
$$

\section{REFERENCES}

1. W. H. Fleming, Stochastic Lagrange Multipliers, in Mathematical Theory of Control, ed. by A. V. Balakrishnan and L. W. Neustadt, Academic Press, New York, 1967. 2. H. P. McKean, Stochastic Integrals, Academic Press, New York, 1969.

3. G. Maruyama, Continuous Markov processes and stochastic equations, Rend. Cir. Mat. Palermo (2), 4 (1955), 48-90.

4. E. J. McShane, Toward a stochastic calculus $I$ and $I I$, Proc. Nat. Acad. Sci., 63 (1969), 275-280 and 1084-1087.

5. - Stochastic integrals and stochastic functional equations, SIAM J. Appl. Math., 17 (1969), 287-306.

6. - Stochastic Calculus and Stochastic Models, to appear.

7. L. S. Pontryagin, V. G. Boltyanskii, R. V. Gamkrelidze, E. F. Mischenko, Thr Mathematical Theory of Optimal Processes (trans. K. N. Trirogoff), Interscience Put lishers, New York, 1962.

8. V. M. Warfield, A Stochastic Maximum Principle, to appear.

Received November 1, 1973. 


\section{PACIFIC JOURNAL OF MATHEMATICS}

EDITORS

RICHARD ARENS (Managing Editor)

University of California

Los Angeles, California 90024

R. A. Beaumont

University of Washington

Seattle, Washington 98105

J. DugundJI

Department of Mathematics

University of Southern California

Los Angeles, California 90007

D. Gilbarg and J. Milgram

Stanford University

Stanford, California 94305

\section{ASSOCIATE EDITORS}

E. F. BECKENBACH

B. H. NeumanN

F. WOLF

K. YOSHIDA

\section{SUPPORTING INSTITUTIONS}

UNIVERSITY OF BRITISH COLUMBIA CALIFORNIA INSTITUTE OF TECHNOLOGY

UNIVERSITY OF CALIFORNIA

MONTANA STATE UNIVERSITY

UNIVERSITY OF NEVADA

NEW MEXICO STATE UNIVERSITY

OREGON STATE UNIVERSITY

UNIVERSITY OF OREGON

OSAKA UNIVERSITY

\author{
UNIVERSITY OF SOUTHERN CALIFORNIA \\ STANFORD UNIVERSITY \\ UNIVERSITY OF TOKYO \\ UNIVERSITY OF UTAH \\ WASHINGTON STATE UNIVERSITY \\ UNIVERSITY OF WASHINGTON \\ $\stackrel{*}{*} \stackrel{*}{*}{ }^{*}{ }^{*}{ }^{2}$ AMERICAN MATHEMATICAL SOCIETY \\ NAVAL WEAPONS CENTER
}




\section{Pacific Journal of Mathematics}

\section{Vol. 52, No. $1 \quad$ January, 1974}

David R. Adams, On the exceptional sets for spaces of potentials ............ 1

Philip Bacon, Axioms for the Čech cohomology of paracompacta ............ 7

Selwyn Ross Caradus, Perturbation theory for generalized Fredholm operators ..... 11

Kuang-Ho Chen, Phragmén-Lindelöf type theorems for a system of nonhomogeneous equations ............................ 17

Frederick Knowles Dashiell, Jr., Isomorphism problems for the Baire classes .......

M. G. Deshpande and V. K. Deshpande, Rings whose proper homomorphic images are right subdirectly irreducible . . . . . . . . . . . . . . . . . . . . . . . . .

Mary Rodriguez Embry, Self adjoint strictly cyclic operator algebras .............

Paul Erdős, On the distribution of numbers of the form $\sigma(n) / n$ and on some related

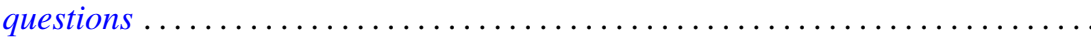

Richard Joseph Fleming and James E. Jamison, Hermitian and adjoint abelian

operators on certain Banach spaces ............................

Stanley P. Gudder and L. Haskins, The center of a poset .................. 85

Richard Howard Herman, Automorphism groups of operator algebras . . . ........

Worthen N. Hunsacker and Somashekhar Amrith Naimpally, Local compactness of families of continuous point-compact relations ....................

Donald Gordon James, On the normal subgroups of integral orthogonal groups ....

Eugene Carlyle Johnsen and Thomas Frederick Storer, Combinatorial structures in

loops. II. Commutative inverse property cyclic neofields of prime-power

order.

Ka-Sing Lau, Extreme operators on Choquet simplexes . . . . . . . . . . . . . . 129

Philip A. Leonard and Kenneth S. Williams, The septic character of 2, 3, 5 and $7 \ldots 143$

Dennis McGavran and Jingyal Pak, On the Nielsen number of a fiber map ........ 149

Stuart Edward Mills, Normed Köthe spaces as intermediate spaces of $L_{1}$ and

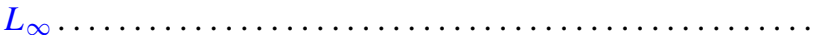

Philip Olin, Free products and elementary equivalence. .

Louis Jackson Ratliff, Jr., Locally quasi-unmixed Noetherian rings and ideals of the principal class.

Seiya Sasao, Homotopy types of spherical fibre spaces over spheres ...

Helga Schirmer, Fixed point sets of polyhedra ...

Kevin James Sharpe, Compatible topologies and continuous irreducible

representations.

Frank Siwiec, On defining a space by a weak base . . . . . . . . . . . . . . . 233

James McLean Sloss, Global reflection for a class of simple closed curves ....... 247

M. V. Subba Rao, On two congruences for primality . .

Raymond D. Terry, Oscillatory properties of a delay differential equation of even

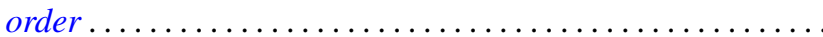

Joseph Dinneen Ward, Chebyshev centers in spaces of continuous functions . .

Robert Breckenridge Warfield, Jr., The uniqueness of elongations of Abelian

groups...

V. M. Warfield, Existence and adjoint theorems for linear stochastic differential

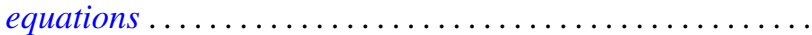

